\title{
Reseña del libro Niños y niñas en situación de calle, de V. Toledo, F. A. De Singlau y S. Abud Rodríguez ${ }^{1}$
}

Laura Noemí Lora²

El libro, escrito por Victor F. Toledo, Silvina V. Abud Rodríguez y Federico de Singlau, nos aporta los resultados de un proyecto de investigación acreditado por el Consejo de Investigación de la Universidad Católica de Salta, titulado "El papel de los actores locales e internacionales en el diseño de políticas públicas destinadas a paliar la situación de los niños y niñas en situación de calle en la Ciudad de Salta", que surgió en el marco de un programa de investigación denominado "La calle como espacio urbano: escenario de experiencia de vida. Niños y adolescentes en riesgo".

En la introducción, Victor Toledo menciona que el principal objetivo del estudio fue el de evaluar las capacidades del municipio de la ciudad de Salta para atender la problemática de la niñez en situación de calle, analizando las políticas públicas implementadas por el Gobierno municipal y el rol que cumplen las organizaciones locales e internacionales en su diseño o implementación (p. 15).

En el capítulo I, titulado "Hacia una conceptualización de las políticas públicas", Toledo desarrolla conceptualmente qué se entiende por política pública, sus definiciones, nociones y alcances. Citando a Ruiz López y a Cárdenas Ayala, dice que una primera aproximación relaciona las políticas públicas con las acciones que los gobiernos encaran para dar respuestas a las demandas de la sociedad. Inmediatamente después aclara que resulta necesario no identificar las políticas públicas con las acciones gubernamentales, dado que en la conceptualización y

\section{Sociedad / reseña descriptivo-crítica}

Citar: Lora, L. N. (2020). Reseña del libro Niños y niñas en situación de calle, de V. Toledo, F. A. De Singlau y S. Abud Rodríguez. En Omnia. Derecho y sociedad, 3 (3), pp. 123-127.

1 Toledo, V.; De Singlau F. A. y Abud Rodríguez, S. (2019). Niños y niñas en situación de calle. Análisis de las políticas públicas en la ciudad de Salta. Salta, EUCASA.

2 Universidad de Buenos Aires (UBA). Abogada por la Facultad de Derecho de la UBA. Doctora en el área Derecho Social; Especialista en Sociología del Derecho y de las Instituciones y Especialista en Administración de Justicia, títulos expedidos por la UBA. Profesora regular en el Área de Ciencias Sociales, especialidad en Sociología, en la Carrera de Abogacía de la Facultad de Derecho de la misma universidad. Docente del curso Metodología de la Investigación Social y Derecho y Sociedad, válido para el Doctorado de dicha Facultad de Derecho. Miembro permanente del Comité Internacional de Investigación en Sociología del Derecho Research Committee on Sociology of Law of ISA 2018-2021. Miembro integrante de la Junta de Gobierno del Comité Internacional de Investigación en Sociología Jurídica (RCSL). Vicepresidenta de la Sociedad Argentina de Sociología Jurídica (SASJU) 2018-2020. Investigadora de la UBA. Directora del Seminario sobre Sociología Jurídica Instituto de Investigaciones Gioja, Facultad de Derecho (UBA) lauranoemilora@derecho.uba.ar 
entramado de una política pública intervienen otros actores, tales como el sector privado, la sociedad civil, la empresa, y otros actores internacionales $u$ organizaciones internacionales.

El autor de este capítulo señala que las políticas públicas conllevan un proceso, y en esta investigación identifica las siguientes etapas: 1) diagnóstico político (problemática); 2) planteo de objetivos; 3) formulación de alternativas de solución; 4) adopción de una de esas alternativas; 5$)$ implementación de la solución; 6) evaluación permanente. Luego de estas etapas se focaliza en tres instancias básicas de las políticas públicas: el problema, la implementación y la evaluación, y les da contenido.

En el capítulo II titulado "¿Niños, niñas y adolescentes "de la calle", "en la calle", o "en situación de calle"? Una aproximación conceptual», el autor, Federico A. de Singlau, señala que en la década de los 90 se intensificó la preocupación que generó el fenómeno de las niñas, niños y adolescentes en situación de calle, y que su visibilidad hace surgir modelos de abordaje y utilización de expresiones en la producción académica latinoamericana, acerca de las cuales destaca lo problemático de su elección, señalando que aunque pueda parecer superficial, no lo es, ya que trae aparejadas implicancias que pueden influir en los resultados de la investigación.

Refiere que en el proyecto se considera "calle" al espacio urbano utilizado por niños, niñas y adolescentes para el despliegue constante o frecuente de sus estrategias de sobrevivencia, que combinan actividad lúdica y económica.

Luego identifica y diferencia los conceptos: niños de la calle o niños callejeros; niños en la calle, niños con experiencia de vida en la calle; niños, niñas y adolescentes en situación de calle.

Sostiene que en América Latina prevaleció la conceptualización escogida por Unicef, se- gún la cual niños de la calle son los habitantes de los grandes centros urbanos cuyos vínculos familiares son muy débiles o nulos, que se desenvuelven en el espacio de la calle, donde desarrollan estrategias con el objeto de obtener ingresos, y encuentran también en ella un ámbito de socialización.

Refiere también que la expresión "niños en la calle" suele asimilarse a niños trabajadores y, por lo tanto, al tipo de actividad que desarrollan. Por este motivo y dado que la investigación pretende alcanzar un objetivo referido a las vivencias de los niños y adolescentes en relación con la calle, este concepto es descartado.

Respecto de la expresión "niños con experiencia de vida en la calle", dice que hace referencia a niños que, si bien tienen experiencia de vida en la calle, en algunos casos prolongada y en otros transitoria, no viven en ella.

La expresión “niños, niñas y adolescentes en situación de calle" es caracterizada a partir de la referencia a distintos especialistas en niñez y adolescencia, entre estos Forselledo (2001), Méndez (2005), Bulgach y Flores (2011). A partir de estos autores, menciona que la expresión es amplia en su significado y que vincula, en general, niños, niñas y adolescentes que viven, deambulan o trabajan en la calle y que provienen de familias en situación de pobreza e indigencia. Son niños, niñas y adolescentes que presentan altos niveles de violencia, con nula o baja atención afectiva y que, en general, han interrumpido la escolaridad; se encuentran expuestos a malos tratos y abusos, y poseen una baja expectativa de vida a causa de los riesgos a los que se exponen a diario.

En la conclusión del capítulo, sostiene que lo más adecuado es el uso de la categoría "niños en situación de calle" para referirse a todos los menores de dieciocho años de edad, sean niñas, niños o adolescentes, sin recurrir a un lenguaje que puede ser estigmatizante o descalificador. 
Además, es un término amplio que engloba diversas situaciones que pueden modificarse.

Mi experiencia en la temática - que se remonta a la década de 1990 y coincide con la época en que su conceptualización, como bien señala de Singlau, se intensificó y ganó espacio en la agenda de temas preocupantes para las sociedades latinoamericanas- permite reafirmar lo sostenido por el autor.

En el capítulo III, "La pobreza y sus múltiples dimensiones", Victor Toledo desarrolla, a través de una visión panorámica, el tema de la pobreza como variable contextual y estructural en la que se inserta la problemática de niños y niñas en situación de calle.

Haciendo referencias a estadísticas obtenidas a través de distintas fuentes, busca responder si los niños y niñas en situación de calle son un "problema" en Salta.

Considera los datos brindados por el Barómetro de la Deuda Social de la Infancia de la Universidad Católica Argentina (UCA), dado que le resultan de utilidad para analizar la evolución de la pobreza y la indigencia por región y por grupos de edad en el país. Observó, sin embargo, la ausencia de algunos datos, especialmente oficiales, para abordar la problemática de la pobreza y la niñez de manera integral.

En el capítulo IV, "Entramado institucional para el abordaje de la problemática de niñez y adolescencia", Toledo indaga sobre el entramado institucional, tanto en el nivel provincial como en el municipal, diseñado para el abordaje de las distintas problemáticas relacionadas con la niñez y la adolescencia. Básicamente, refleja las estructuras gubernamentales, sus organigramas y competencias, entre las que se destacan la elaboración de normas, programas vinculados a la protección integral, cuidado integral, diseño, coordinación y ejecución de políticas sociales, capacitación, comunicación, inclusión de la comunidad, monitoreo, inclu- sive la concreción de convenios con Unicef. También se hace referencia a la relevancia que adquiere el Poder Judicial, por la estrecha relación que tiene con la problemática en cuestión. Se afirma que este constituye una de las fuentes más relevantes de datos estadísticos para la elaboración de políticas públicas.

El autor realiza una crítica a los informes elaborados por las instituciones administrativas, dado que no contienen información completa ni integral, y atribuye esto, como posible factor, a la falta de implementación completa de la Ley 26061, Ley de Protección Integral de Niñas Niños y Adolescentes. No comparto esta afirmación, ya que el autor describe muy detalladamente la descentralización administrativa que se produjo tanto a nivel nacional, provincial y municipal, y que debería, dentro de sus funciones, producir información necesaria para las políticas públicas porque forma parte de las competencias que fueron enunciadas detalladamente.

En el capítulo $V$ de la obra, bajo el título "La inversión social", Victor Toledo expone las dimensiones relativas a las erogaciones presupuestarias, cifras y análisis sobre la inversión social por parte de la nación, de la provincia y del municipio de Salta. Con esa información aborda el tema de los recursos financieros destinados a la problemática de niñez y adolescencia, aportando datos cuantitativos. Respecto del análisis presupuestario, destaca una vez más lo segmentado de la información y su difícil acceso especialmente en el período 2013-2016. Concluye diciendo que no se muestra en detalle el destino de los fondos, motivo por lo cual no puede identificar los recursos destinados a paliar la situación de niños y niñas en situación de calle. También señala la fuerte dependencia que existe de parte de las jurisdicciones locales con respecto a los fondos federales. Destaca también que la mayoría 
de los recursos del Ministerio de Derechos Humanos, del gasto social, a grandes rasgos están orientados a la cuestión alimentaria. A nivel municipal, gran parte del presupuesto está destinado a los salarios del personal. Por último, afirma que Salta fue una de las provincias que más incrementó el gasto social destinado a la niñez $y$, sin embargo, presenta una de las tasas de mortalidad infantil más altas del país, por lo que advierte la necesidad de reforzar el control de la gestión.

El último capítulo, titulado "Niños y Niñas en situación de calle en Salta", está escrito con la participación, como coautores, de Toledo, Abud Rodríguez y De Singlau. Los tres integrantes del equipo describen el trabajo de campo de la investigación. Analizan información de dependencias gubernamentales, así como del rol que tienen las ONG en relación con los casos de los niños y niñas en situación de calle. Mencionan el detalle, no menor, respecto de la conceptualización de la situación de calle, concepto que, al ser tan abarcativo, hace que esta no sea visibilizada como tal en tanto el niño o la niña no vivan "en" la calle.

Acerca de las situaciones descriptas en los capítulos anteriores, refieren que son difíciles de comprender, como por ejemplo, el hecho de la constatación de planes o actividades que no responden a necesidades o demandas, y que no hay carencias que las justifiquen ni peticiones que las expliquen $y$, sin embargo, han sido elaboradas e implementadas. También señalan que hay necesidades que quedan sin atender, porque no derivan de demandas explícitas, como es el caso de los niños y niñas en situación de calle.

Los autores mencionan que debido a las dificultades en el acceso a los datos, han recurrido en busca de información a la División de Protección al Menor y la Familia de la Policía de la provincia de Salta, a la que consultaron por derivación de los Defensores de Menores e Incapaces. Detallan las instituciones hacia las cuales los casos de menores son derivados con finalidades de "guarda" y que dependen de organismos oficiales, y también de la labor intensa de las ONG. A través de entrevistas que realizaron en algunas de estas instituciones, pudieron relevar información, aunque esta no se focaliza en la temática objeto de estudio del equipo debido fundamentalmente a que no es visibilizada como problemática.

Las conclusiones finales son un recuento de las conclusiones provisorias que fueron obteniendo en cada capítulo del libro. Son relevantes los obstáculos que se mencionan con respecto al acceso a la información estadística, más precisamente respecto de la denegación de datos que en principio no se sabe si están elaborados. En efecto, los autores afirman "... los organismos públicos, tanto municipales como provinciales, se abstuvieron de facilitar los datos estadísticos que solicitamos de forma reiterada, lo cual impidió que este trabajo reflejara dicha información de manera integral o completa..." (p. 146).

Los autores expresan que existe una falta de adecuación de las instancias políticas de la provincia de Salta a los compromisos internacionales asumidos por la Argentina en materia de derechos de la niñez y la adolescencia, y que el tema de la niñez en situación de calle no es considerado un problema por el municipio de la capital salteña.

Con relación a las entrevistas que realizaron en las instituciones, plantean resultados en relación con la falta de recursos humanos capacitados, dado que se trata de una problemática multidimensional, y por el hecho de que muchas de las instituciones están vinculadas a las fuerzas de seguridad.

Respecto del trabajo que realizan las ONG, observan que este tiene baja repercusión en 
la elaboración de políticas públicas. A nivel provincial, los convenios de cooperación, especialmente con Unicef, están orientados a la capacitación, pero no se pudo mensurar su impacto en la formulación de políticas públicas. En el ámbito de la gestión, se advierte falta de articulación en el nivel endógeno y falta de control externo, en el nivel exógeno.

En cuanto a lo macroeconómico, sobre la base de los resultados que aporta el Observatorio de la Deuda Social de la UCA, afirman que Salta se encuentra en un contexto de alto índice de pobreza y que ello repercute en la situación de niñas y niños, a la par que son elevados los índices de riesgo y vulnerabilidad.
Esta vulnerabilidad de los niños y sus familias dificulta las demandas efectivas para que estas se vuelquen luego en políticas públicas.

Antes de finalizar, quiero felicitar a los autores por la edición y publicación del libro colectivo, ya que visibiliza la problemática a través de experiencias del equipo de trabajo que fueron reseñadas aquí. El desafío de la realización de diagnósticos a partir de los cuales pensar y diseñar políticas públicas para los niños y niñas en situación de calle está explícito. Sin lugar a dudas, mucha información disponible en este libro se convierte en un antecedente valioso para elaborar políticas públicas de manera urgente. 